\title{
CONFIANÇA, APRENDIZAGEM E CONHECIMENTO NOS RELACIONAMENTOS INTERORGANIZACIONAIS: DIAGNÓSTICO E ANÁLISE DOS AVANÇOS SOBRE O TEMA
}

\author{
Juliano Nunes Alves \\ admjuliano@yahoo.com.br \\ Universidade de Cruz Alta - Cruz Alta, RS / Brasil \\ Breno Augusto Diniz Pereira \\ brenodpereira@gmail.com \\ Universidade Federal de Santa Maria - Santa Maria, RS / Brasil \\ Taís de Andrade \\ tais0206@gmail.com \\ Universidade Federal de Santa Maria - Santa Maria, RS / Brasil \\ Eliete dos Reis \\ elietedosreis@gmail.com \\ Universidade Federal de Santa Maria - Santa Maria, RS / Brasil
}

Recebido em 06/10/2011

Aprovado em 18/12/2012

Disponibilizado em 01/12/2013

Avaliado pelo sistema double blind review

Revista Eletrônica de Administração

Editor: Luís Felipe Nascimento

ISSN 1413-2311 (versão on-line)

Editada pela Escola de Administração da Universidade Federal do Rio Grande do Sul.

Periodicidade: Quadrimestral

Sistema requerido: Adobe Acrobat Reader.

\section{RESUMO}

Compreender o estado de evolução de um tema é necessário, em determinado momento, para o processo de evolução da ciência, a fim de que se ordene periodicamente o conjunto de informações e resultados já obtidos, ordenação que permita indicação das possibilidades de integração de diferentes perspectivas, aparentemente autônomas, a identificação de duplicações ou contradições, e a determinação de lacunas e vieses. Dessa forma, busca-se verificar a agenda da pesquisa atual sobre os três construtos principais: confiança, aprendizagem e conhecimento, estabelecida por diversas publicações em periódicos internacionais (101 periódicos). Esses periódicos foram extraídos do Qualis da área de Administração, Contabilidade e Turismo. Foram avaliados primeiramente os títulos e resumos de todos os artigos publicados e posteriormente lidos todos os artigos encontrados sobre o tema foco do estudo. Foram encontrados e lidos 55 artigos publicados sobre o tema em seis anos de análise (2004 a 2009). Os principais resultados estão comprovação da importância que os temas confiança, aprendizagem e conhecimento, independentes ou relacionados contribuindo para uma melhor velocidade, a flexibilidade e agilidade em competitividade frente as variações do ambiente onde as redes estão inseridas. 
Confiança, aprendizagem e conhecimento nos relacionamentos interorganizacionais: diagnóstico e análise dos avanços sobre o tema

Palavras-Chave: Relacionamentos Interorganizacionais; Conhecimento; Aprendizagem; Confiança.

\title{
CONFIDENCE, LEARNING AND KNOWLEDGE IN THE INTER- ORGANIZATIONAL RELATIONSHIPS: DIAGNOSIS AND ANALYSIS OF THE DEVELOPMENTS ON THE THEME
}

\begin{abstract}
Understanding the state of evolution of a theme is necessary in certain moment for the process of science evolution, in order for the set of information and results already obtained to be periodically ordered, ordination which allows indicating the possibilities of integration of different perspectives, apparently autonomous, identifying duplications or contradictions and determining gaps and deviations. Thus, it is intended to verify the agenda of the current research on the three constructs, established by several publications in international journals (101 journals). These journals were extracted from Qualis in the areas of Business Administration, Counting and Tourism. First, the titles and abstracts of all published articles were evaluated and afterwards all the articles about the theme in study were read. Within six years of analysis (2004 to 2009), 55 articles published on the theme were found and read. The main results are proof of the importance that the topics trust, learning and knowledge, independent or related contributing to improved speed, flexibility and agility in competitiveness against changes in environment where networks are located.
\end{abstract}

Keywords: Inter-Organizational Relationships; Knowledge; Learning, Confidence

\section{CONFIANZA, APRENDIZAJE Y CONOCIMIENTO EN RELACIONES INTERORGANIZACIONALES: DIAGNÓSTICO Y ANÁLISIS DE LOS PROGRESOS SOBRE EL TEMA}

\begin{abstract}
RESUMEN
Conocer el estado de la evolución de un tema es necesario, en algún momento, para el proceso de evolución de la ciencia, por lo que periódicamente se ordene el conjunto de la información y los resultados ya obtenidos, categorización que permita indicación de las posibilidades de la integración de diferentes perspectivas, aparentemente autónoma, la identificación de duplicaciones y contradicciones, vacíos y oblicuidades. De este modo, se busca comprobar la programación de la investigación actual sobre las tres construcciones, creado por varias publicaciones en revistas internacionales (101 revistas). Estas revistas fueron tomados del Qualis de zona de la Administración, Contabilidad y Turismo. En primer lugar, evaluaron los títulos y los resúmenes de todos los artículos publicados y después se leyó todos los artículos sobre el foco objeto del estudio. Ha se encontrado 55 artículos publicados sobre el tema de análisis en seis años (2004 a 2009). Los principales resultados son una prueba de la importancia que el fideicomiso temas, el aprendizaje y el conocimiento,
\end{abstract}


independientes o relacionados que contribuye a mejorar la velocidad, la flexibilidad y agilidad de competitividad frente a cambios en el entorno en que las redes se encuentran.

Palabras Clave: Las Relaciones Interorganizacionales; Conocimiento; Aprendizaje; Confianza.

\section{INTRODUÇÃO}

A investigação sobre redes de empresas é multifacetada, ou seja, existem várias abordagens e uma literatura substancial (BORGATTI; FOSTER, 2003; MÖLLER; RAJALA, 2007). Todavia, na literatura, a necessidade e a motivação para a criação de redes organizacionais têm sido explicada através de duas perspectivas: a teoria dos custos de transação (TCT) e da teoria baseada em recursos (TBR). Especialmente a teoria baseada em recursos enfatiza o papel de colaboração e de redes como uma fonte de recursos complementares, conhecimento e aprendizagem, ao passo que na teoria dos custos de transação a explicação é para que a rede seja construída sobre uma melhor eficiência com o intuito de reduzir os custos através de suas operações. Assim, as razões gerais para a cooperação e o trabalho em rede incluem, por exemplo, a maior disponibilidade de recursos e conhecimentos, a concentração em competências, a possibilidade de criação de novos conhecimentos e aprendizagem, o acesso a novas oportunidades (mercados, tecnologias, diferenciação do produto) e inovação, as economias de escala e de escopo ou partilha de custos e riscos (NOOTEBOOM, 1999).

Relacionamentos interorganizacionais (redes) estão se tornando cada vez mais comuns e complexos, assim os seus impactos sobre as empresas se multiplicam. O desenvolvimento da cooperação começou com alianças que se formaram entre duas organizações individuais que estavam dispostas a cooperar devido as suas necessidades e capacidades complementares. As alianças estratégicas têm sido um importante tema de estudo para a gestão estratégica e para os negócios internacionais, onde uma das formas de alianças amplamente pesquisada são as joint ventures (INKPEN, 1998). Nesse ponto, a capacidade de cooperação dos parceiros vem sendo amplamente abordada (INKPEN; CROSSAN, 1995).

Nos relacionamentos interorganizacionais podem ser considerados tanto o ator como o contexto, dependendo do foco e nível de análise. Por um lado, uma rede é uma entidade que pode ser analisada de forma independente e pode ser vista como um ator. Por outro lado, uma rede é sempre constituída por entidades menores, como organizações, e pode ser vista como um ambiente circundante, ou seja, passivo no contexto. Esta abordagem destaca o papel do indivíduo nas organizações no âmbito dos relacionamentos interorganizacionais, enquanto 
Confiança, aprendizagem e conhecimento nos relacionamentos interorganizacionais: diagnóstico e análise dos avanços sobre o tema

que a postura anterior destaca a holística, ou seja, a natureza sistêmica desse relacionamento. A dupla natureza do conceito tem também implicações para estudar fenômenos relacionados à rede, tais como a confiança, aprendizagem e conhecimento.

Diante dessas perspectivas, há uma grande mobilização acadêmica e empresarial com o intuito de estudar o comportamento entre organizações. Buscando verificar o que está sendo publicado na academia ao longo dos últimos anos sobre relacionamentos interorganizacionais no que tange a confiança, aprendizagem e conhecimento, este estudo pretende servir como referência para pesquisas posteriores sobre o tema. Serão avaliados os últimos avanços, os principais focos de investigação e se tais estudos estão em declínio ou em desenvolvimento. Dentro dessa perspectiva, o desenvolvimento do presente estudo se deu por meio de pesquisa bibliográfica e análise dos artigos publicados nos últimos seis anos nos periódicos da Qualis da Capes para a área de Administração, Contabilidade e Turismo.

\section{REFERENCIAL TEÓRICO}

\subsection{Redes Interorganizacionais}

Rede, como conceito, possui uma infinidade de definições; é usado de modo diferente de acordo com a perspectiva. Pode ser definida como uma resposta estratégica da empresa às pressões dinâmicas ambientais, proporcionando um incentivo para delegar funções corporativas ou favorecer parceiros especializados em colaborações e alianças.

Há vários modos de exibição de uma organização em rede. Do ponto de vista comportamental, é um padrão de relações sociais ao longo de um conjunto de pessoas, posições, grupos ou organizações (SAILER, 1978). Do ponto de vista estratégico, é considerada como os acordos de longo prazo entre os propósitos distintos, mas relacionados que permitem que as empresas envolvidas ganhar ou manter uma vantagem competitiva frente as que não estão em redes (JARILO, 1988). Seja qual for a opinião atribuída a uma organização em rede, todas elas têm em comum a necessidade de gerir o conhecimento de seus membros para que os seus objetivos possam ser alcançados com o esforço menor do que seria atingido individualmente.

\subsection{Fatores de Sucesso nas relações interorganizacionais}


Juliano Nunes Alves \& Breno Augusto Diniz Pereira

Existem diversos fatores que contribuem para a formação e gestão das redes. O presente estudo, todavia, limita-se a identificar os trabalhos publicados sobre confiança, conhecimento e aprendizagem.

\subsubsection{Confiança}

A confiança é um conceito multidimensional que foi revelado para conter várias dimensões que compõem o construto (ZARVANDI; ZARVANDI, 2012). Dentre as dimensões McCole argumenta que "a literatura revela que a confiança permeia os relacionamentos por meio de dez dimensões, que são: disponibilidade, competência, consistência, singularidade, imparcialidade, integridade, lealdade, transparência, cumprimento promessa e receptividade" (1992, p.39).

Essas dimensões defendidas por McCole (2002, p. 39) anteriormente foram também categorizadas por Swan et. al. (1985) e Swan e Trawick (1987) onde abordam que a confiança é baseada em cinco dimensões, a saber: confiabilidade, honestidade, competência, orientação e facilidade. Ou seja, existem diversas formas de dimensionar a confiança reforçando a ideia de que é um conceito multidimensional, porém, ao longo do tempo, embora o volume de negócios de trabalhadores e gestores possam ocorrer em diversas dimensões, o processo é complexo devido dificuldade de ser operacionalizada essas dimensões tanto a nível interorganizacional quanto interpessoal.

Corroborando no estudo de Henriques et. al. (2010) verifica-se que existe uma dificuldade nessa conceitualização e operacionalização do conceito de confiança pelo fato de que advém da afirmação frequente de que " ...a confiança é um conceito complexo, que foi sendo analisado através de uma pluralidade de disciplinas..." (GULATI; NICKERSON, 2008 , p. 2) e que, por isso, “....as definições de confiança variam de um contexto para outro e também tendo em conta a disciplina de referência.” (IBBOTT; O’KEEFE, 2004, p.133).

No âmbito interorganizacional como aborda Tacconi et. al. (2011) a confiança referese à "extensão com que os membros de uma organização retêm uma confiança coletiva orientada em direção a outra organização" (ZAHEER; HARRIS, 2006, p.170). Existe ainda uma distinção muito tênue entre confiança interorganizacional e confiança interpessoal, pois as duas estão presentes nas organizações. Nesse sentido, Barney e Hansen (1994) e recentemente Kremer et. al., (2012) afirmam que a confiança exerce uma importante influência na concretização dos relacionamentos interorganizacionais cooperativos e que esta 
Confiança, aprendizagem e conhecimento nos relacionamentos interorganizacionais: diagnóstico e análise dos avanços sobre o tema

depende principalmente da confiança interpessoal entre indivíduos que ligam os setores de fronteira das organizações, ou seja, aqueles que se relacionam entre organizações.

\subsubsection{Aprendizagem Organizacional e Interorganizacional}

Os estudos sobre aprendizagem em nível organizacional e interorganizacional têm apontado para várias perspectivas teóricas. No que se refere ao âmbito organizacional, essas perspectivas podem ser caracterizadas sob uma visão técnica e social da aprendizagem (EASTERBY-SMITH e ARAÚJO, 2001). A visão técnica diz respeito ao processamento eficaz, à interpretação e à resposta das informações tanto internas quanto externas às organizações. Já a visão social focaliza o modo pelo qual os indivíduos atribuem significados às experiências de trabalho. Nessa perspectiva, "a aprendizagem organizacional é algo que emerge de interações sociais, normalmente no ambiente natural de trabalho" (EASTERBYSMITH; ARAÚJO, 2001 p. 19).

Ainda, de acordo com Ruiz-Mercader, Merono-Cerdan e Sabater-Sánchez (2006), a aprendizagem organizacional é definida como o processo que aumenta o conhecimento da organização e de seus membros através da compreensão, interpretação e assimilação de informações tácitas e explícitas. No entender de Dodgson (1993), a aprendizagem organizacional é a capacidade que as organizações têm de adquirir e organizar conhecimentos e rotinas em torno das suas atividades dentro da cultura organizacional, bem como adaptar e desenvolver a eficiência organizacional através da melhoria do uso de habilidades gerais e de suas forças de trabalho.

$\mathrm{Na}$ esfera da aprendizagem interorganizacional, existe uma gama de conceitos que pressupõe o envolvimento de fatores como cultura, estrutura, tecnologia e capacidade absortiva (LEVINSON e ASAHI, 1995). Dessa forma, grande parte das pesquisas sobre aprendizagem interorganizacional tem se concentrado no papel da capacidade absortiva, ou seja, na habilidade de uma empresa assimilar e utilizar novos conhecimentos externos (LANE e LUBATKIN, 1998).

Sob a ótica de Cohen e Levinthal (1990), a capacidade absortiva ocorre em grande parte em função do nível de conhecimento prévio relacionado. No nível mais elementar este conhecimento prévio inclui habilidades básicas ou até mesmo uma linguagem em comum, e pode também incluir o conhecimento dos mais recentes desenvolvimentos científicos ou tecnológicos em um determinado campo. Assim, o conhecimento prévio relacionado confere 
uma capacidade de reconhecer o valor de novas informações, assimilá-las e aplicá-las para fins comerciais (COHEN e LEVINTHAL, 1990).

De acordo com Levinson e Asahi (1995), a capacidade absortiva é a fundamentação para a aprendizagem interorganizacional e transferência de conhecimento entre organizações (SHILDT; KEIL; MAULA, 2012). Para os autores, existem quatro passos que devem ser observados no processo de aprendizagem interorganizacional: as organizações devem desenvolver a capacidade de identificar novos conhecimentos; devem interpretar e transferir novos conhecimentos; devem utilizar o conhecimento ajustando o comportamento para alcançar os resultados pretendidos e; devem institucionalizar o conhecimento refletindo sobre o que está acontecendo.

Ainda, no entender de Larsson et al. (1998), a aprendizagem interorganizacional pode ser vista como a aquisição coletiva de conhecimento entre um conjunto de organizações. Nesse contexto, as alianças estratégicas desenvolvem conhecimento coletivo por construir e modificar seu ambiente interorganizacional, regras de trabalho e preferências (LARSSON et al., 1998). Além disso, a aprendizagem acontece naturalmente por meio do compartilhamento de diferentes conhecimentos e experiências entre as organizações que participam de algum tipo de aliança estratégica.

No caso das redes interorganizacionais, segundo Owen-Smith e Powell (2004), a aprendizagem desempenha um papel chave, agindo como guardiã das regras e práticas das organizações participantes de uma rede, resultando no desenvolvimento de lógicas dominantes que passam a definir o ritmo organizacional dentro da rede. Dessa forma, a aprendizagem interorganizacional se substancia por incluir sinergia de aprendizado ou efeito de interação entre as organizações.

\subsubsection{Conhecimento}

O conhecimento assume uma centralidade nos estudos interorganizacionais, sendo considerado um recurso importante para garantir a vantagem competitiva das organizações. As pesquisas sobre o tema evidenciam sua complexidade e multiplicidade de abordagens, destacando-se as visões distintas do discurso normativo e interpretativo.

Conforme Schultze; Ledner (2002), na visão do discurso normativo predomina a natureza racional do conhecimento, ressaltando-se a possibilidade de seu gerenciamento e controle, tendo como foco o processo e as práticas de trabalho. Por outro lado, o discurso interpretativo enfatiza o conhecimento associado às práticas organizacionais e ao princípio do 
Confiança, aprendizagem e conhecimento nos relacionamentos interorganizacionais: diagnóstico e análise dos avanços sobre o tema

conhecimento socialmente construído por meio da interação entre os indivíduos, o qual contribui com a transformação organizacional. Sob este enfoque, o conhecimento está ligado diretamente a estruturas sociais, o que dificulta sua gestão (ZAIDMAN; BROCK, 2009).

Em consonância com o discurso interpretativo, Nonaka (1994) advoga que o conhecimento pode ser definido como uma crença verdadeira justificada que aumenta a capacidade dos indivíduos para uma ação efetiva em uma organização, sendo que sua partilha pode ocorrer por meio do fornecimento ou recebimento de informações, know-how e feedback (CUMMINGS, 2004). Nonaka; Takehuchi (1997) destacam ainda, que o processo pelo qual as organizações criam conhecimento está na mobilização e conversão do conhecimento tácito. Assim, o processo de conversão do conhecimento organizacional inicia no nível individual, movendo-se para a dimensão grupal e para o nível interorganizacional (KROGH; NONAKA; RECHSTEINER, 2012).

Nas redes interorganizacionais aspectos importantes para analisar esse processo referem-se ao compartilhamento e transferência de conhecimentos (ČERNAITÉ; SUDINTAITE், 2012). Sob este enfoque, Dyer; Singh (1998) destacam que as rotinas de compartilhamento de conhecimento, consideradas como um padrão regular de interações entre organizações que permite a transferência, combinação ou criação de conhecimento especializado, exercem um papel relevante, podendo contribuir para o melhor desempenho da empresa (DYER; HATCH, 2006). Alguns estudos também têm demonstrado a importância do compartilhamento de conhecimento, ao permitir que as organizações melhorem o desempenho nas inovações e reduzam os esforços redundantes em aprendizagem (CALANTONE et. al., 2002; SCARBROUGH, 2003).

Em relação a transferência de conhecimentos Easterby-Smith; Lyles; Tsant (2008) destacam que uma empresa pode significativamente melhorar os seus conhecimentos e capacidades inovadoras, valendo-se das habilidades dos outros, através da transferência de conhecimento entre as empresas. Nesse sentido, Lane; Lubatkin (1998) afirmam que a aprendizagem interativa pode contribuir para o desenvolvimento de capacidades singulares e valiosas, através da transferência de conhecimentos articuláveis e tácitos. Entretanto, a transferência de conhecimento é um fenômeno complexo e, na prática, muitas vezes pode não ocorrer (EASTERBY-SMITH; LYLES; TSANT, 2008).

Ainda, mesmo considerando a relevância da aprendizagem e do compartilhamento e transferência de novos conhecimentos, percebe-se que há algumas divergências entre os autores quanto a distinção e interação entre os construtos conhecimento e aprendizagem. Por 
um lado, alguns autores destacam que as abordagens sobre aprendizagem carecem da visão de que o desenvolvimento do conhecimento constitui aprendizado e não desenvolveram uma visão ampla sobre sua definição e interação (NONAKA; TAKEHUSHI, 1997; KROGH; NONAKA; RECHSTEINER, 2012). Por outro lado, existem algumas críticas de que as abordagens sobre criação do conhecimento, mesmo defendendo as contribuições do conhecimento tácito e da aprendizagem por meio da experiência, não mencionam a relação entre ação e conhecimento, instigando os debates acerca das temáticas (EASTERBY-SMITH et al., 2000).

Contudo, mesmo não havendo um consenso sobre as definições e interações entre conhecimento e aprendizagem, percebe-se que esses construtos compartilham conceitos subjacentes e complementares, embora ainda estejam usando uma linguagem diferente para expressar essas questões (EASTERBY-SMITH et al., 2000). Considerando essa perspectiva, neste trabalho buscou-se definir conhecimento como um conjunto de aprendizagens acumuladas de um indivíduo, organização ou rede, que lhe permitem exercer atividades rotineiras e resolver possíveis problemas.

\section{MÉTODO DE TRABALHO}

A metodologia utilizada para o desenvolvimento deste trabalho foi pesquisa bibliográfica com objetivo descritivo, a fim de proporcionar maior familiaridade com o problema, aprimorando as concepções já existentes (GIL, 1996). Trata-se de uma pesquisa teórica quanto a sua natureza e bibliográfica quanto aos procedimentos técnicos. Consiste em identificar nos periódicos internacionais o estágio em que se encontram os estudos sobre confiança, aprendizagem e conhecimento e nos relacionamentos interorganizacionais, bem como, apresentar o quanto evoluiu a procura por esse tema e seus principais pesquisadores e achados no decorrer dos últimos seis anos.

No entanto, é necessário destacar que a metodologia da pesquisa bibliográfica apresenta a limitação decorrente da amplitude e qualidade das fontes de consulta. Nesse artigo, esta limitação também decorre da escolha da base de consulta restrita a periódicos internacionais; porém, reconhecidamente importantes como veículo de divulgação dos avanços acadêmicos na área da administração. É importante salientar que o presente estudo não tem o objetivo de esgotar o tema, mas de servir como referência para estudos mais avançados sobre ele.

REAd | Porto Alegre - Edição 76 - N 3 - setembro/dezembro 2013 - p. 709-737 
Confiança, aprendizagem e conhecimento nos relacionamentos interorganizacionais: diagnóstico e análise dos avanços sobre o tema

Para este trabalho, foram selecionados todos os artigos publicados nos periódicos internacionais do Qualis da Capes na área de Administração, Contabilidade e Turismo - no período compreendido entre 2004 e 2009 - sobre relacionamentos interorganizacionais, com corte para os estudos de confiança, aprendizagem e conhecimento nesses relacionamentos.

A escolha tanto do período quanto dos periódicos foi intencional. O Qualis Capes é o ranking relevante na área acadêmica de Administração no Brasil, agrupando o maior número de pesquisas recentes da área, principalmente as realizadas nos principais centros de estudo do mundo.

Visando a atender ao objetivo de identificar e avaliar a teoria sobre relacionamentos interorganizacionais através de três construtos principais: confiança, aprendizagem e conhecimento, foram utilizados como critério de avaliação para cada um dos artigos presentes na amostra selecionada os seguintes itens: a quantidade de publicações/ano sobre o tema, as abordagens mais utilizadas, a classificação no qualis ao longo dos anos, os periódicos, os países mais estudados, as teorias mais utilizadas, os autores e os principais resultados ao longo do período estudado.

O total de artigos selecionados foi dividido igualitariamente entre dois pesquisadores da área para codificação e análise. Todos os artigos encontrados foram analisados conjuntamente por ambos os pesquisadores, de forma a permitir equalização de procedimentos e a minimizar possíveis diferenças de percepção.

A partir desse momento procedeu-se a codificação e análise de cada artigo de acordo com os atributos apresentados anteriormente. Para isso, num primeiro momento, os artigos foram classificados pela avaliação no Qualis. Em seguida, todos os artigos foram categorizados, por ano, de acordo com a sua classificação: A1; A2; B1; B2; B3; B4 e B5. A etapa seguinte foi dividida em três:

1. identificou-se os temas mais estudados e os países que publicaram sobre o tema buscando verificar quais os temas mais pesquisados e a evolução dos países ao longo do período avaliado;

2. identificou-se as principais teorias e autores mais utilizados pelos pesquisadores ao longo do período estudado, buscando apresentar as teorias mais reconhecidas e utilizadas no meio acadêmico. Classificou-se também se o tipo de pesquisa era teórica ou empírica; 
3. Por fim, foram elencados os focos de estudos sobre relacionamentos interorganizacionais no decorrer do período pesquisado e os resultados encontrados nos trabalhos pesquisados.

Para a obtenção do conjunto de informações e artigos, realizou-se num primeiro momento a tabulação dos dados e, posteriormente, a leitura de todos os artigos, de forma a identificar os quesitos relativos à metodologia, principais resultados, teorias utilizadas e o local onde foi realizado o estudo. Estes elementos não costumam ser abordados nos resumos dos trabalhos e, em muitos casos, foram identificados pelos métodos estatísticos descritos e o momento em que foram realizados - caracterizando, assim, a ocorrência desses procedimentos ou não. Essa estratégia de trabalho também permitiu complementar dados faltantes e levou a uma maior confiabilidade na codificação, tabulação e análise dos dados uma vez que foram frequentes equívocos como, por exemplo, a alusão à aplicação da pesquisa em um lugar, no resumo, e a caracterização de outro, na seção análise dos resultados.

\section{APRESENTAÇÃO DOS RESULTADOS}

A Tabela 1 mostra a distribuição do número total de artigos sobre relacionamentos interorganizacionais apresentados nos periódicos internacionais. A parte superior da coluna ilustra o total de publicações sobre relacionamentos interorganizacionais, enquanto a parte inferior ilustra o total de trabalhos com relação a abordagem confiança, conhecimento e aprendizagem. Do total de estudos publicados nesses anos (171 trabalhos), 55 artigos abordam confiança, aprendizagem interorganizacional ou confiança, como pode ser observado na Tabela 1.

Tabela 1 - Distribuição por ano da produção relacionada ao tema

\begin{tabular}{|c|c|c|}
\hline Ano & $\begin{array}{l}\text { Trabalhos publicados sobre relacionamentos } \\
\text { interorganizacionais }\end{array}$ & $\begin{array}{l}\text { Trabalhos sobre a área de confiança, } \\
\text { conhecimento e aprendizagem }\end{array}$ \\
\hline 2004 & 10 & 0 \\
\hline 2005 & 22 & 9 \\
\hline 2006 & 29 & 9 \\
\hline 2007 & 36 & 11 \\
\hline 2008 & 31 & 13 \\
\hline 2009 & 43 & 13 \\
\hline TOTAL & 171 & 55 \\
\hline
\end{tabular}


Confiança, aprendizagem e conhecimento nos relacionamentos interorganizacionais: diagnóstico e análise dos avanços sobre o tema

Desta verificação, é possível constatar, apesar de um crescimento do número de publicações sobre relacionamento interorganizacionais, uma estabilidade quanto ao número de publicações relacionadas a confiança, aprendizagem interorganizacional e/ou conhecimento ao longo dos últimos anos, excetuando-se o ano de 2004; em que não foram localizados artigos sobre o argumento.

A Tabela 2 ilustra esse montante em percentual no período estudado. A faixa percentual de publicação sobre relacionamentos interorganizacionais com foco nos temas confiança, aprendizagem e conhecimento varia entre $30,23 \%$ a $41,94 \%$, não apresentando uma variação regular que poderia ser classificada como crescente ou decrescente. Em relação ao número total de estudos realizados sobre relacionamentos interorganizacionais, 32,16\% representam as pesquisas sobre confiança, aprendizagem e conhecimento.

Tabela 2 - Percentual de publicação a propósito de confiança, conhecimento ou aprendizagem, do total de publicações sobre relacionamentos interorganizacionais

\begin{tabular}{cc}
\hline Ano & \% em relação ao número total de trabalhos publicados \\
\hline $\mathbf{2 0 0 4}$ & $0 \%$ \\
\hline $\mathbf{2 0 0 5}$ & $40,91 \%$ \\
\hline $\mathbf{2 0 0 6}$ & $31,03 \%$ \\
\hline $\mathbf{2 0 0 7}$ & $30,56 \%$ \\
\hline $\mathbf{2 0 0 8}$ & $41,94 \%$ \\
\hline $\mathbf{2 0 0 9}$ & $30,23 \%$ \\
\hline TOTAL & $\mathbf{3 2 , 1 6 \%}$ \\
\hline
\end{tabular}

Fonte: Elaborada pelos autores

A Tabela 3 sintetiza as abordagens utilizadas nos artigos foco deste estudo. Percebe-se que em média $72,73 \%$ dos estudos publicados sobre o assunto foram de natureza empírica. Todavia é possível verificar um crescimento dos estudos teóricos a partir de 2006 em contrapartida ao declínio da abordagem empírica a partir desse mesmo ano.

Tabela 3 - Tipo de abordagem dos trabalhos

\begin{tabular}{cccccc}
\hline ANO & Total de trabalhos sobre & \multicolumn{2}{c}{ Abordagem teórica } & \multicolumn{2}{c}{ Abordagem empírica } \\
\cline { 3 - 5 } & $\begin{array}{c}\text { o tema nos periódicos } \\
\text { internacionais }\end{array}$ & Artigos & $\%$ & Artigos & $\%$ \\
2004 & 0 & 0 & $0 \%$ & 0 & $0 \%$ \\
2005 & 9 & 3 & $33,33 \%$ & 6 & $66,67 \%$ \\
2006 & 9 & 0 & $0 \%$ & 9 & $100 \%$ \\
2007 & 11 & 3 & $27,27 \%$ & 8 & $72,73 \%$ \\
2008 & 13 & 4 & $30,77 \%$ & 9 & $69,23 \%$ \\
2009 & 13 & 5 & $38,46 \%$ & 8 & $61,54 \%$ \\
TOTAL & $\mathbf{5 5}$ & $\mathbf{1 5}$ & $\mathbf{2 7 , 2 7 \%}$ & $\mathbf{4 0}$ & $\mathbf{7 2 , 7 3 \%}$ \\
\hline
\end{tabular}


Em relação aos trabalhos com abordagem empírica, percebeu-se que os mesmos referem-se a estudos analíticos, baseados em experiências em seu contexto local. Pode-se identificar também que houve um aumento no volume de pesquisas bibliográficas nos últimos três anos, sendo que em 2008 e 2009 se constatou a mesma quantidade de estudos descritivos e bibliográficos. Verifica-se assim, que existe um equilíbrio entre as metodologias do estudo, pois vinte e três deles foram quantitativos e vinte e dois foram qualitativos e cinco foram qualitativos/quantitativos e apenas quatro foram teóricos, sendo um experimento.

Ao que se refere à participação dos artigos nos periódicos internacionais, apresenta-se na Tabela 4, em ordem de volume de publicação total, os periódicos a classificação no qualis do respectivo periódico e como as publicações se distribuíram entre os anos de 2005 e 2009; visto que em 2004 não foram localizadas pesquisas sobre os temas investigados.

Tabela 4 - Distribuição por periódico

\begin{tabular}{|l|c|c|c|c|c|c|c|}
\hline Periódico & Qualis & $\mathbf{2 0 0 5}$ & $\mathbf{2 0 0 6}$ & $\mathbf{2 0 0 7}$ & $\mathbf{2 0 0 8}$ & $\mathbf{2 0 0 9}$ & TOTAL \\
\hline Human Relations & $\mathrm{A} 1$ & 3 & 1 & 2 & & 1 & $\mathbf{7}$ \\
\hline Management Decision & $\mathrm{A} 1$ & & & 3 & 1 & 2 & $\mathbf{6}$ \\
\hline Strategic Management Journal & $\mathrm{A} 1$ & 1 & 1 & & 1 & 3 & $\mathbf{6}$ \\
\hline Expert Systems with Applications & $\mathrm{A} 1$ & & 2 & & 3 & & $\mathbf{5}$ \\
\hline International Journal of Information Management & $\mathrm{A} 1$ & & 2 & & 1 & 1 & $\mathbf{4}$ \\
\hline Journal of Management Studies & $\mathrm{A} 1$ & 1 & & & 2 & 1 & $\mathbf{4}$ \\
\hline European Journal of Operational Research & $\mathrm{A} 1$ & & 2 & & & 1 & $\mathbf{3}$ \\
\hline Organization Studies & $\mathrm{A} 1$ & 2 & & & & 1 & $\mathbf{3}$ \\
\hline Journal of International Management & $\mathrm{A} 2$ & 1 & & 2 & 2 & 2 & $\mathbf{7}$ \\
\hline Science Technology; Society & $\mathrm{A} 2$ & & 1 & 1 & & & $\mathbf{2}$ \\
\hline Journal of Technology Management; Innovation & $\mathrm{B} 1$ & & & & 2 & & $\mathbf{2}$ \\
\hline Management Research News & $\mathrm{B} 1$ & & & 1 & & 1 & $\mathbf{2}$ \\
\hline Outros & & 1 & & 2 & 1 & & $\mathbf{4}$ \\
\hline TOTAL & & $\mathbf{9}$ & $\mathbf{9}$ & $\mathbf{1 1}$ & $\mathbf{1 3}$ & $\mathbf{1 3}$ & $\mathbf{5 5}$ \\
\hline
\end{tabular}

Fonte: Elaborado pelos autores

Pôde-se constatar um maior número de publicações nos periódicos Human Relations e Journal of International Management nos últimos seis anos. Destaca-se, no entanto, o volume no Journal of International Management nos anos de 2007; 2008 e 2009 e o crescimento do Management Decision nesse mesmo período, no que se refere às publicações sobre confiança, conhecimento e aprendizagem em relacionamentos interorganizacionais, com seis publicações cada. Por outro lado, pode-se verificar uma redução dos estudos no periódico Human Relations, onde passou de três publicações em 2005 para nenhuma em 2008 e apenas uma em 2009.

REAd | Porto Alegre - Edição 76 - N 3 - setembro/dezembro 2013 - p. 709-737 
Confiança, aprendizagem e conhecimento nos relacionamentos interorganizacionais: diagnóstico e análise dos avanços sobre o tema

Quanto ao crescimento dos estudos evidenciou-se que em 2008 e 2009 publicaram-se 13 trabalhos por ano. Somados, 2008 e 2009 representam aproximadamente 50\% das publicações do período avaliado, demonstrando a atualidade dos assuntos investigados.

Ainda que as pesquisas sobre confiança, conhecimento e aprendizagem em relacionamentos organizacionais sejam recentes, a importância do tema é constatada pelo nível de qualificação dos periódicos em que os artigos são publicados. A maior parte das publicações são em periódicos de classificação Qualis/Capes A1 (56,25\%) e A2 (25\%), isso representando $81,25 \%$ das publicações totais.

Quanto às palavras chaves mais utilizadas pelos estudos destaca-se o termo conhecimento, presente em (56\%) das publicações sobre o tema, seguido de confiança (33\%) e aprendizagem (24\%), como ilustra a Tabela 5. Tais resultados demonstram que os estudos analisados destacam a relevância do conhecimento nos relacionamentos interorganizacionais, corroborando as perspectivas de Easterby-Smith; Lyles; Tsang (2008) de que as pesquisas empíricas da última década tem enfatizado a importância do compartilhamento e transferência de conhecimento entre empresas. Da mesma forma, Schildt; Keil; Maula (2012) destacam que a compreensão empírica dos fluxos de conhecimentos em redes tem sido ampliada em pesquisas recentes, aliadas a busca de melhor entendimento das mudanças nas relações interorganizacionais ao longo do tempo.

Tabela 5 - Palavras-chave nas publicações sobre o tema

\begin{tabular}{lcc}
\hline Conhecimento & $\mathbf{3 1}$ & $\mathbf{5 6 \%}$ \\
\hline Confiança & 18 & $33 \%$ \\
Aprendizagem & 13 & $24 \%$ \\
Inovação & 12 & $22 \%$ \\
Recursos & 7 & $13 \%$ \\
Competências & 6 & $11 \%$ \\
\hline
\end{tabular}

Fonte: Elaborado pelos autores

Além do aumento de interesse e relevância dada ao tema, como pode ser observado pelas análises anteriores, é interessante perceber que as pesquisas estão sendo conduzidas em diversos países. Na Tabela 6 apresenta-se os resultados da participação dos países nas publicações.

Tabela 6 - Países pesquisados sobre o tema

\begin{tabular}{ll}
\hline País & Total Publicações \\
\hline Estados Unidos & 14 \\
China & 11 \\
\hline
\end{tabular}

REAd | Porto Alegre - Edição 76 - N 3 - setembro/dezembro 2013 - p. 709-737 
Juliano Nunes Alves \& Breno Augusto Diniz Pereira

\begin{tabular}{lc}
\hline Reino Unido & 4 \\
Brasil & 3 \\
\hline Espanha & 3 \\
\hline Austrália & 2 \\
\hline Bélgica & 2 \\
Outros & 16 \\
Total & $\mathbf{5 5}$ \\
\hline & Fonte: Elaborado pelos autores
\end{tabular}

Destacam-se Estados Unidos (25,45\%) e China (20,00\%) quanto ao número de publicações sobre o tema pesquisado. Ainda que mencionados na Tabela 4 somente os países com maior volume de artigos publicados, ressalta-se que nos últimos seis anos (2004-2009) os temas confiança, conhecimento e aprendizagem em relacionamentos interorganizacionais foram estudados em 23 países, sendo que as publicações sobre relacionamentos interorganizacionais sem tal delimitação foram verificadas em 42 países.

Conforme observado, há um interesse da comunidade acadêmica mundial sobre o tema. Os autores estão distribuídos em diversos países. É de se constatar também o aspecto territorial dos estudos (regionalidade), onde poucos são os estudos que abordam países ou até mesmo continentes diferentes. Com isso, torna-se necessário proporcionar aos estudos realizados sobre aprendizagem, conhecimento e confiança nos relacionamentos interorganizacionais uma possibilidade de aprofundamento e de comparação. No Quadro 2 sintetiza-se os autores mais utilizados como base teoria e suas respectivas contribuições teóricas.

Quadro 02 - Autores utilizados como base teórica nos trabalhos publicados (continua)

\begin{tabular}{|c|c|}
\hline Autor & Contribuições teóricas \\
\hline $\begin{array}{l}\text { GRANOVETTER, } \\
1973\end{array}$ & Relações interpessoais, em geral, vêm em três variedades: forte, fraco ou ausente. \\
\hline WILLIAMSON, 1975 & $\begin{array}{l}\text { Os formatos organizacionais (ou estruturas de "governança") - firma, mercado ou } \\
\text { redes, por exemplo - são resultado da busca de minimização dos custos de } \\
\text { transação por parte dos agentes econômicos. }\end{array}$ \\
\hline $\begin{array}{l}\text { TEECE, 1976; 1986; } \\
\text { 1996, TEECE; GARY; } \\
\text { AMY, } 1997\end{array}$ & $\begin{array}{l}\text { Foca seus estudos em Capacidade dinâmica a qual é definida como "a capacidade } \\
\text { da empresa de integrar, construir e reconfigurar competências internas e externas } \\
\text { para enfrentar ambientes em rápida mudança". }\end{array}$ \\
\hline $\begin{array}{l}\text { COHEN; } \\
\text { LEVINTHAL, } 1990\end{array}$ & $\begin{array}{l}\text { Quando uma empresa deseja adquirir e utilizar conhecimentos alheios à sua } \\
\text { atividade em curso, deve desenvolver a capacidade de absorção. Dois fatores } \\
\text { afetam os incentivos da empresa para a aprendizagem - a quantidade de } \\
\text { conhecimento e dificuldade (custo) de aprendizagem. } \\
\text { (continuação) }\end{array}$ \\
\hline HAMEL, 1991 & $\begin{array}{l}\text { A colaboração pode proporcionar uma oportunidade para um parceiro para } \\
\text { internalizar as habilidades dos outros, e assim melhorar a sua posição dentro e } \\
\text { fora da aliança. }\end{array}$ \\
\hline
\end{tabular}

REAd | Porto Alegre - Edição 76 - N 3 - setembro/dezembro 2013 - p. 709-737 
Confiança, aprendizagem e conhecimento nos relacionamentos interorganizacionais: diagnóstico e análise dos avanços sobre o tema

\begin{tabular}{|c|c|}
\hline GULATI, 1995 & $\begin{array}{l}\text { Aborda em seus estudos que a confiança interorganizacional representa a } \\
\text { expectativa de uma organização que outra empresa não irá agir de forma } \\
\text { oportunista quando se relacionar com essa organização. }\end{array}$ \\
\hline ZAHEER et. al., 1995 & $\begin{array}{l}\text { Que a ligação entre confiança interpessoal e interorganizacional é baseada em } \\
\text { processos de institucionalização. a confiança interpessoal e interorganizacional } \\
\text { estão relacionados, mas distintos construtos, e desempenham papéis diferentes no } \\
\text { que respeita aos processos de negociação e desempenho. }\end{array}$ \\
\hline DAS; TENG, 1996 & $\begin{array}{l}\text { Eles Apresentam três tipos de controle: produção, comportamentais e sociais. } \\
\text { Controle de saída é a avaliação do desempenho do parceiro. Controlar o } \\
\text { comportamento é o de assegurar que o processo é adequado. O controle social } \\
\text { (controle ou informal) se concentra no desenvolvimento de valores, crenças e } \\
\text { objetivos entre os membros. }\end{array}$ \\
\hline UZZI, 1997 & $\begin{array}{l}\text { Aborda o conceito de embeddedness o qual amplia a tipologia típica das teorias } \\
\text { de câmbio para incluir uma nova categorização por motivos (racionalidade } \\
\text { emergente) e de racionalidade (a racionalidade expert). }\end{array}$ \\
\hline DYER et. al., 1998 & $\begin{array}{l}\text { Em seus estudos confiança é um antecedente importante para cooperação } \\
\text { interorganizacional e de eficiência econômica. }\end{array}$ \\
\hline GHERARDI, 1999 & $\begin{array}{l}\text { A maneira de se estudar aprendizagem nas organizações e a incorporação de } \\
\text { elementos como as metáforas e o poder para compreender melhor os fenômenos } \\
\text { sociais referentes aos temas aprendizagem e geração de conhecimento. }\end{array}$ \\
\hline HANSEN, 1999 & $\begin{array}{l}\text { A transferência de conhecimentos e sinergias em empresas de várias unidades } \\
\text { devem perseguir novas perspectivas que combinam os conceitos de conexões de } \\
\text { rede e de relacionamento no conteúdo do conhecimento. }\end{array}$ \\
\hline ARIÑO et. al., 2001 & $\begin{array}{l}\text { Considera que a qualidade relacional tanto como um contributo para o sucesso do } \\
\text { empreendimento, e uma saída das interações entre os parceiros. }\end{array}$ \\
\hline $\begin{array}{l}\text { DAVENPORT; } \\
\text { PRUSAK, } 2003\end{array}$ & $\begin{array}{l}\text { O conhecimento pode ser comparado a um sistema vivo, que cresce e se modifica } \\
\text { à medida que interage com o meio ambiente. }\end{array}$ \\
\hline
\end{tabular}

Fonte: Elaborado pelos autores

A Quadro 3 demonstra os principais resultados encontrados nos estudos sobre confiança interorganizacional, divididos em quatro fases principais: compreensão do construto, importância do construto; início/formação da rede e evolução do relacionamentos interorganizacionais.

Quadro 3 - Principais resultados apontados pelos estudos sobre o Construto Confiança Interorganizacional, entre os anos de 2004 a 2009.

(continua)

\begin{tabular}{|c|c|c|}
\hline $\begin{array}{l}\text { Fases do } \\
\text { Processo }\end{array}$ & Resultados & Autores \\
\hline $\begin{array}{c}\text { Compreensão } \\
\text { do construto }\end{array}$ & $\begin{array}{l}\text { 1. A confiança se refere aos comportamentos anti-sociais } \\
\text { indiretamente, através de sentimentos de apego à organização e seus } \\
\text { membros; } \\
\text { 2. É plausível que a violação da confiança em um nível pode levar à } \\
\text { violação de confiança em outro nível, independente da capacidade de um } \\
\text { nível para realizar um determinado esforço de reparar ou não. } \\
\text { 3. Modelo que ilustra como a dependência de confiança, tanto } \\
\text { afetivo e cognitivo varia à medida do desenvolvimento da rede; } \\
\text { 4. A confiança no parceiro está ligada aos novos conhecimentos, } \\
\text { habilidades, e competências adquiridas através da aliança. }\end{array}$ & $\begin{array}{l}\text { Muthusamy; White, } \\
\text { 2005; Thau; } \\
\text { Crossley; Bennett; } \\
\text { Sczesny, 2007; } \\
\text { Smith; Lohrke, 2008; } \\
\text { Janowicz-Panjaitan; } \\
\text { Krishnan, 2009 }\end{array}$ \\
\hline & & (continuação) \\
\hline $\begin{array}{l}\text { Importância } \\
\text { para o sucesso } \\
\text { da rede }\end{array}$ & $\begin{array}{l}\text { 1. O nível de confiança vai influenciar a eficácia da rede; } \\
2 . \quad \text { Confiança e aprendizagem cooperativa surgiram como fatores } \\
\text { críticos que afetam o sucesso das alianças estratégicas; }\end{array}$ & $\begin{array}{l}\text { De Wever; Martens; } \\
\text { Vandenbempt, 2005; } \\
\text { Mellat-Parast; }\end{array}$ \\
\hline
\end{tabular}

REAd | Porto Alegre - Edição 76 - N 3 - setembro/dezembro 2013 - p. 709-737 


\begin{tabular}{|c|c|c|}
\hline & $\begin{array}{l}\text { 3. O relacionamento é mais forte quando o nível de confiança é } \\
\text { maior. }\end{array}$ & $\begin{array}{l}\text { Digman, 2007; Wai- } \\
\text { Kit Ng; Lau; Nyaw, } \\
2007\end{array}$ \\
\hline $\begin{array}{l}\text { Início/formação } \\
\text { da rede }\end{array}$ & $\begin{array}{l}\text { 1. Quando a confiança não estava presente, representantes das } \\
\text { organizações tentaram erguer a confiança sob a forma de contratos ou de } \\
\text { governança para a ponte. }\end{array}$ & $\begin{array}{l}\text { Stephens; Fulk; } \\
\text { Monge, } 2009\end{array}$ \\
\hline $\begin{array}{l}\text { Evolução da } \\
\text { rede }\end{array}$ & $\begin{array}{l}\text { 1. Longo prazo tem um efeito positivo sobre a amizade, lealdade, } \\
\text { confiança e compromisso; } \\
2 . \quad \text { Quanto mais tempo tem um relacionamento interorganizacional, } \\
\text { mais forte é a associação entre competência e confiança na aquisição de } \\
\text { conhecimento. }\end{array}$ & $\begin{array}{l}\text { Pesämaa; Hair Jr, } \\
\text { 2007; Lui, } 2009\end{array}$ \\
\hline
\end{tabular}

Fonte: Elaborado pelos Autores

Para o construto confiança observa-se uma formalização do processo. Apesar de vários estudos ainda se centraram na compreensão do tema, estudos mais recentes já ousam tentar aplicar tais conceitos em trabalhos de cunho mais prático. Todavia os resultados ainda são bastante limitados, carecendo de estudos mais focados que estabeleçam uma relação mais concreta entre confiança e o sucesso dos relacionamentos interorganizacionais. Por exemplo, questões como: 1) Com o aumento da confiança diminui os mecanismos de controle? 2) Maior confiança possibilita maiores ganhos? 3) Em redes de muitas empresas é possível estabelecer mecanismos de sucesso para se obter a confiança?

Independentemente do enfoque teórico dado a compreensão do construto confiança em relacionamentos interorganizacionais, um ponto central está refletido em todos eles: a importância da confiança para a perpetuação dos relacionamentos interorganizacionais. Isso reforça que independente da perspectiva ou dos objetivos do relacionamento, a confiança deve ser buscada de forma primordial. Ela é a chave para a criação, manutenção e principalmente para a evolução dos relacionamentos cooperativos.

Quadro 4 - Principais resultados apontados pelos estudos sobre o Construto Aprendizagem Interorganizacional, entre os anos de 2004 a 2009. (continua)

\begin{tabular}{|c|c|c|}
\hline $\begin{array}{l}\text { Fases do } \\
\text { Processo }\end{array}$ & Resultados & Autores \\
\hline $\begin{array}{c}\text { Compreensão } \\
\text { do construto }\end{array}$ & $\begin{array}{l}\text { 1. Redes de conhecimento fornecem um veículo importante } \\
\text { para o indivíduo e aprendizagem em grupo; } \\
\text { 2. Aprendizagem se desenvolve a partir de um conjunto } \\
\text { complexo de interações ou inter-relações entre sujeitos e objetos } \\
\text { como um evento em curso de se relacionar e responder; } \\
\text { 3. Quando fortemente arraigadas normas e valores } \\
\text { enfatizando existir aprendizagem de nível superior, as } \\
\text { desvantagens do volume de negócios disfuncionais são } \\
\text { provavelmente reduzidas; }\end{array}$ & $\begin{array}{l}\text { Walker; Chiristenson, } \\
\text { 2005; Kupers, 2008; } \\
\text { Guidice; Heames; Wang, } \\
2009\end{array}$ \\
\hline & & \\
\hline $\begin{array}{l}\text { Importância } \\
\text { para o sucesso } \\
\text { da rede }\end{array}$ & $\begin{array}{l}\text { A aprendizagem individual e organizacional tem mostrado } \\
\text { efeitos significativos e positivos sobre o desempenho } \\
\text { organizacional; }\end{array}$ & $\begin{array}{l}\text { Ruiz-Mercader; Merono- } \\
\text { Cerdan; Sabater- } \\
\text { Sánchez, } 2006\end{array}$ \\
\hline
\end{tabular}

REAd | Porto Alegre - Edição 76 - N 3 - setembro/dezembro 2013 - p. 709-737 
Confiança, aprendizagem e conhecimento nos relacionamentos interorganizacionais: diagnóstico e análise dos avanços sobre o tema

Fonte: Elaborado pelos autores

Aprendizagem tem sido reconhecida na literatura como um processo importante para a gestão dos relacionamentos cooperativos, bem como para a criação de novos benefícios. Os resultados deste trabalho demonstram que o tema ainda é pouco estudado e principalmente compreendido pela comunidade científica. A utilização de diferentes perspectivas da literatura de aprendizagem em redes permite compreender a profundidade e visualizar o fenômeno de maneira a tornar possível avaliar e integrar as idéias centrais de cada abordagem.

Além disso, a divisão do Quadro 4 em fases do processo (compreensão e importância do tema) permite enfatizar as características gerais encontradas nos trabalhos analisados e com isso expor e avaliar a importância relativa e adequação dos pontos de vista tanto em linhas do nível de rede quanto para o de rede de aprendizagem. Com base na literatura exposta, pode-se perceber que concepções teóricas foram as que mais enfatizaram o construto.

Essa combinação de diferentes pontos de vista apresentados pelos estudos sobre o tema não deixa de ser adequado pelo fato da área estudada e possibilita nesta pesquisa estender com a compilação das teorias existentes conseguindo com isso um maior nível de detalhe a respeito de algumas perspectivas teóricas.

Quadro 5 - Principais resultados apontados pelos estudos sobre o Construto Conhecimento Interorganizacional, entre os anos de 2004 a 2009.

\begin{tabular}{|c|c|c|}
\hline $\begin{array}{l}\text { Fases do } \\
\text { Processo }\end{array}$ & Resultados & Autores \\
\hline $\begin{array}{l}\text { Compreensão } \\
\text { do construto }\end{array}$ & $\begin{array}{l}\text { 1. O conhecimento do produto, o conhecimento do cliente e o } \\
\text { conhecimento gerencial são complementares; } \\
\text { 2. Fluxos de conhecimento entre profissionais de diferentes grupos } \\
\text { permitem que os indivíduos estabeleçam as margens operacionais } \\
\text { para autonomia; } \\
\text { 3. A velocidade de transferência de conhecimento e poder de } \\
\text { influência dos indivíduos desempenham um papel dominante no } \\
\text { processo de transferência de conhecimento; } \\
\text { 4. Estratégias comuns auxiliam a rede a gerenciar o conhecimento; } \\
\text { 5. Não há evidência de que inter e intraorganizational transferência de } \\
\text { conhecimento sejam distintas; } \\
\text { 6. Aplicação sistemática de mecanismos de transferência de } \\
\text { conhecimento pode superar a viscosidade e ambiguidade causal de } \\
\text { novos conhecimentos; } \\
\text { 7. Os esforços de criação de conhecimento atual e conhecimento prévio } \\
\text { são substituíveis na produção de conhecimento colaborativo. }\end{array}$ & $\begin{array}{c}\text { Tanriverdi; } \\
\text { Venkatraman, } \\
\text { 2005; Tagliaventi; } \\
\text { Mattarelli, 2006; } \\
\text { Tang; Mu; } \\
\text { Maclachlan, 2008; } \\
\text { Easterby-Smith; } \\
\text { Lyles; Tsang, } \\
\text { 2008; Inkpen, } \\
\text { 2008 } \\
\text { (contínuação) }\end{array}$ \\
\hline $\begin{array}{c}\text { Importância } \\
\text { para o sucesso } \\
\text { da rede }\end{array}$ & $\begin{array}{l}\text { 1. Empiricamente, os recursos de conhecimento da rede possuem uma } \\
\text { influência significativa no desempenho da empresa; }\end{array}$ & Dyer; Hatch, 2006 \\
\hline $\begin{array}{c}\text { Início/formaçã } \\
\text { o da rede }\end{array}$ & $\begin{array}{l}\text { 1. O primeiro tipo de fluxo de conhecimento é intencional do ponto } \\
\text { de vista de ambas as redes, enquanto o segundo pode não ser }\end{array}$ & $\begin{array}{l}\text { Zhao; Anand; } \\
\text { Mitchell, 2005; }\end{array}$ \\
\hline
\end{tabular}

REAd | Porto Alegre - Edição 76 - N 3 - setembro/dezembro 2013 - p. 709-737 


\begin{tabular}{|c|c|c|}
\hline & $\begin{array}{l}\text { desejável ou intencional da multinacional para a rede; } \\
\text { Investimento prévio na criação de conhecimentos, quer através da } \\
\text { experiência ou esforços anteriores que determinam a capacidade de } \\
\text { absorção, leva a melhores resultados por causa do custo, infra- } \\
\text { estrutura, conhecimento negativo, a vantagem de acelerar o } \\
\text { desenvolvimento, etc; } \\
\text { 3. A complementaridade entre os parceiros da aliança tem impacto } \\
\text { significativo sobre a capacidade de uma empresa para realizar ação } \\
\text { conjunta com seus parceiros e clientes e no acesso ao } \\
\text { conhecimento. }\end{array}$ & $\begin{array}{c}\text { Samaddar; } \\
\text { Kadiyala, 2006; } \\
\text { Schreiner; Kale; } \\
\text { Corsten, } 2009\end{array}$ \\
\hline $\begin{array}{l}\text { Evolução da } \\
\text { rede }\end{array}$ & $\begin{array}{l}\text { 1. O resultado é melhor comunicado quando há uma ampla aplicação } \\
\text { do conhecimento organizacional que reforça a concepção e } \\
\text { exploração de trabalho cooperativo; } \\
\text { 2. Interdependência e complexidade do conhecimento, provocada } \\
\text { pela especialização, teve um efeito importante na interação das } \\
\text { relações entre a coordenação e partilha de conhecimento; } \\
\text { 3. Há um impacto positivo e significativo da cultura organizacional } \\
\text { na aquisição de conhecimento; } \\
\text { 4. A utilização de mecanismos pessoais de comunicação é favorável } \\
\text { em situações de baixa distância geográfica, tais como comunicação } \\
\text { face-a-face pode permitir uma transferência de conhecimentos mais } \\
\text { suave; } \\
\text { 5. Informalidade é mais eficaz na transferência de conhecimento do } \\
\text { que na estrutura da hierarquia. }\end{array}$ & $\begin{array}{c}\text { Coakes; Coakes; } \\
\text { Rosenberg, 2008; } \\
\text { Willem; Buelens, } \\
\text { 2009; Park; } \\
\text { Whitelock; } \\
\text { Giroud, 2009; } \\
\text { Ambos; Ambos, } \\
2009\end{array}$ \\
\hline
\end{tabular}
Fonte: Elaborado pelos autores

Avaliando os trabalhos encontrados na literatura (Quadro 5) pode-se caracterizar o construto conhecimento como sendo o mais abordado. O conhecimento está relacionado diretamente a resolução de problemas e principalmente a obtenção de novos benefícios (inovações) em processos cooperativos. Ou seja, o surgimento do processo cooperativo busca a criação de capacidades que individualmente os membros não possuem. Dessa forma, o entendimento e formalização do conhecimento dos membros são imprescindíveis para o sucesso de um empreendimento. Estudos recentes como Willem; Buelens (2009); Park; Whitelock; Giroud (2009) e Ambos; Ambos (2009) já demonstram maneiras eficazes de criação de conhecimento em relacionamentos cooperativos, o que possibilita a esse formato organizacional perspectivas sustentáveis.

Com os resultados apresentados pode-se perceber que confiança, conhecimento e aprendizagem são fatores determinantes para $o$ sucesso dos relacionamentos interorganizacionais. Além disso, verifica-se que a grande maioria dos estudos apresentados buscam modos de identificar ou sugerir formas de aumentar a capacidade competitiva e o desempenho das organizações através dos relacionamentos que mantém com outras organizações.

\section{CONSIDERAÇÕES FINAIS}


Confiança, aprendizagem e conhecimento nos relacionamentos interorganizacionais: diagnóstico e análise dos avanços sobre o tema

A análise dos artigos possibilitou estabelecer um panorama das pesquisas publicadas nos últimos seis anos, em periódicos internacionais classificados no Qualis da Capes da Administração, Contabilidade e Turismo. Com isso, foi possível atingir o objetivo proposto do trabalho de analisar como os temas confiança, conhecimento e aprendizagem interorganizacional estão sendo estudados na área de relacionamentos inteorganizacionais e, por meio da análise, se há evolução nesses estudos.

Observou-se que, nos periódicos internacionais, os primeiros estudos foram encontrados a partir de 2005; não sendo localizado nenhum estudo no ano de 2004 que abordasse confiança, conhecimento e aprendizagem; de 2005 a 2009; as publicações sobre este assunto obtiveram crescimento.

Em relação aos resultados da análise dos artigos, foi possível ressaltar algumas constatações: 1) o assunto é recente e uma das aplicações realizadas pelos artigos analisados são as propostas de modelos teóricos e a-aplicações empírica de teorias existentes sobre o tema; 2) as pesquisas são conduzidas, em sua grande maioria, por abordagens inicialmente qualitativas e, mais recentemente, quantitativas; 3) gestão e troca de conhecimentos são atualmente o principal interesse das pesquisas; 4) os antecedentes que levaram a demonstrar os aspectos de conhecimento e confiança foram os estudos sobre formação das redes, que valorizava a necessidade de acesso a recursos materiais e imateriais, além da busca por fatores de sustentabilidade nas relações; 5) os estudos na Ásia e Europa evidenciaram crescimento; 6) há uma lacuna de estudos na África e na América do Sul; 7) os temas estudados representam $32 \%$ dos estudos sobre relacionamentos interorganizacionais nos periódicos internacionais.

A compreensão de diferentes construtos para atividades de gestão das redes são importantes, porque por meio da aprendizagem, confiança e conhecimento pode-se melhorar a velocidade, a flexibilidade e o tempo de chegada ao mercado com produtos/serviços diferenciados e com isso melhorar a competitividade das redes e das organizações inseridas nela. A compreensão do tema, combinado com suas implicações em rede pode facilitar a aplicação de técnicas e ferramentas eficazes. Nos estudos que retratavam pesquisas empíricas em redes, especialmente as práticas relacionadas ao aprendizado, exibições de memória organizacional, conhecimento tácito e explícito, utilização e exploração da aprendizagem foram enfatizadas e facilmente identificadas.

Por outro lado, observou-se que as práticas relacionadas a outros pontos de vista da aprendizagem, tais como capacidade absortiva ou capacidades dinâmicas são difíceis de identificar, o que pode ser devido à complexidade dos conceitos e as dificuldades em 
relacioná-los à prática. Em geral, aprendizagem não está sendo sistematicamente apoiada nas operações rotineiras, especialmente em nível das redes, mas sim a importância da aprendizagem para a competitividade e as estratégias de futuro do negócio.

Há inúmeros fatores que podem afetar o fenômeno da aprendizagem em ambiente interorganizacionais, tais como: a) como ela ocorre; b) quais os objetivos da colaboração; c) a saída desejada da colaboração; d) o tipo de benefícios criado e qual a fase do processo de evolução da rede; e) o estágio das relações entre os membros da rede, e, f) a definição de papéis e responsabilidades. Por fim sugere-se que sejam analisados confiança, conhecimento e aprendizagem ao longo do tempo em propostas longitudinais para que consiga avanços do conhecimento sobre os temas nos relacionamentos interorganizacionais.

\section{REFERÊNCIAS}

AMBOS, T. C.; AMBOS, B. The impact of distance on knowledge transfer effectiveness in multinational corporations. Journal of International Management, v. 15, p. 1-14, 2009.

ARGOTE, L.; INGRAM, P. Knowledge transfer: A basis for competitive advantage in firms. Organizational Behavior and Human Decision Processes, v. 82, p. 150-169, 2000.

ARIÑO, A.; DE LA TORRE, J.; RING, P.S. Relational quality: management trust in corporate alliances, California Management Review, v.44, n.1, p.109-31, 2001.

BARNEY, J. B.; HANSEN, M. H. Trustworthiness as a Source of Competitive Advantage. Strategic Management Journal, v. 15, n. s1, p. 175-190, 1994. ISSN 1097-0266.

BORGATTI, S. B.; FOSTER, P. C. The Network Paradigm in Organizational Research: A Review; Typology, Journal of Management, v. 29, n. 6, p. 991-1013, 2003.

CALANTONE, R.J.; CAVUSGIL, S.T.; ZHAO, Y. Learning orientation, firm innovation capability, firm performance, Industrial Marketing Management, v. 31, n. 6, p. 515-524, 2002.

ČERNAITĖ, K.; SUDINTAITÉ, L. Does context matter? Study of knowledge sharing in European Institute of Innovation and Technology. 2012. $71 \mathrm{f}$. Tese (Managing People Knowledge and Change) - Lund University, Lund, Suécia, 2012 
Confiança, aprendizagem e conhecimento nos relacionamentos interorganizacionais: diagnóstico e análise dos avanços sobre o tema

COAKES, J.; COAKES, E.; ROSENBERG, D., Co-operative work practices and knowledge sharing issues: a comparison of viewpoints, International Journal of Information Management, v. 28, n. 1, p. 12-25, 2008.

COHEN W; LEVINTHAL L. Absorptive capacity: a new perspective on learning and innovation. Administrative Science Quarterly, v.35, n.1, p.128-152, 1990.

CUMMINGS, J. N. Work groups, structural diversity, and knowledge sharing in a global organization. Management Science, v. 50, n. 3, p. 352-364, 2004.

DAS, T.K.; TENG, B.S. Risk types and inter-firm alliance structures. Journal of Management Studies, v.33, n.6, p.827-843, 1996.

DAVENPORT, T. H.; PRUSAK, L. Conhecimento empresarial. 11. Rio de Janeiro: Elsevier, 2003.

DE WEVER, S.; MARTENS, R.; VANDENBEMPT K. The impact of trust on strategic resource acquisition through interorganizational networks: Towards a conceptual model. Human Relations, v. 58, n. 12, p. 1523-1543, 2005.

DEAN, G. Organisational Knowledge: Capturing its diversity and sharing its power, Anais da Conferência Australasian Libraries in the Emergency Sector (ALIES), 2009.

DYER, J. H.; HATCH, N. W. Relation-specific capabilities and barriers to knowledge transfers: creating advantage through network relationships. Strategic Management Journal. Chichester, mai, 2006, v 27, p. 701.

DODGSON, M. Organizational learning: a review of some literatures. Organization Studies, v.14 n.3 p.357-394, 1993.

EASTERBY-SMITH, M., GROSSAN, M., NICOLINI, D. Organizational Learning: debates past, present and future. Journal of Management Studies, v.. 37, n..6, p. 783-796, 2000.

EASTERBY-SMITH, M., ARAÚJO, L. Aprendizagem organizacional: oportunidades e debates atuais. In: EASTERBY-SMITH, M.; BURGOYNE, J.; ARAÚJO, L. (orgs.). Aprendizagem organizacional e organização de aprendizagem: desenvolvimento na teoria e na prática. São Paulo: Atlas, 2001. 
EASTERBY-SMITH, M.; LYLES, M.; TSANG, E. Inter-organizational knowledge transfer: current issues and future prospects, Journal of Management Studies, v. 45, n. 4, p 661-674, 2008.

ESTIVALETE, V. F. B.; PEDROZO, E. Á.; BEGNIS, H. S. M. O processo de aprendizagem em redes horizontais do elo varejista do agronegócio: uma análise sob a perspectiva das estratégias, dos métodos e dos estágios evolutivos. REAd. Rev. eletrôn. adm. (Porto Alegre), Porto Alegre, v. 18, n. 1, Apr. 2012.

GHERARDI, S. A symbolic approach to competence development. Human Resource Development International, v. 2, n. 4, 1999.

GIL, A. C. Como Elaborar Projetos de Pesquisa. São Paulo: Atlas, 1996.

GRANOVETTER, M. The strength of weak ties. American Journal of Sociology, v. 78, n. 6, p. 1360-80, May 1973.

GUIDICE, R. M; HEAMES, J. T; SHENG W. The Indirect relationship between organizational-level knowledge worker turnover and innovation. An integrated application of related literature. Disponível em: www.emeraldinsight.com/0969-6474.htm . Acesso em: 18 mar. 2010.

GULATI, R. Social structure; alliance formation patterns: a longitudinal analysis. Administrative Science Quarterly, Ann Harbor, v. 40, p. 619-652, 1995.

GULATI, R.; NICKERSON, J. A. Interorganizational trust, governance choice, and exchange performance. Organization Science, v. 19, n. 5, p. 688-708, 2008.

HAMEL, G. Competition for Competence and Inter-Partner Learning within International Strategic Alliances. Strategic Management Journal, v. 12(Summer), p. 83-103, 1991.

HENRIQUES, M.; RIBEIRO, Ó.; VELOSO, A.; KEATING; J.. O estudo da confiança interorganizational nos últimos cinco anos (2004 -2008) - Revisão Crítica, Anais do VII Simpósio Nacional de Investigação em Psicologia, Portugal, 2010.

HANSEN, M. T . The search-transfer problem: The role of weak ties in sharing knowledge across organization subunits. Administrative Science Quarterly. v. 44, n. 1, p. 82-111, 1999.

REAd | Porto Alegre - Edição 76 - N 3 - setembro/dezembro 2013 - p. 709-737 
Confiança, aprendizagem e conhecimento nos relacionamentos interorganizacionais: diagnóstico e análise dos avanços sobre o tema

IBBOTT, C. J.; O’KEEFE, R. M. Trust, planning and benefits in a global interorganizational system. Infomation Systems Journal, v. 14, p. 131-152, 2004.

INKPEN, A. C.; CROSSAN, M. M. Believing is seeing: joint ventures; organization learning, Journal of Management Studies, v. 32, n. 5, p. 595-618, 1995.

A.C. Learning; knowledge acquisition through international strategic alliances, Academy of Management Executive, v. 12, n. 4, p. 69-80, 1998.

JANOWICZ-PANJAITAN, M.K.,; NOORDERHAVEN, N.G. Trust, calculation; interorganizational learning of tacit knowledge: An organizational roles perspective. Organization Studies, v. 30, n. 10, p. 1021-1044, 2009.

JARILO, J. C. On strategic networks. Strategic Management Journal, v. 9; p. 31-41, 1988.

KNIGHT, L. Network Learning: Exploring Learning by Interorganizational Networks. Human Relations, New York, v. 55, n. 4, p. 427-454, 2002.

L.; PYE, A. Network Learning: an empirically-derived model of learning by groups of organizations. Human Relations, v. 58, n. 3, p. 369-392, 2005.

KREMER, A. M.; AKAHOSHI, W. B.; CAVALHEIRO, R. T.. Uma aplicação empirica da análise de redes sociais informais em uma associação de produtores rurais. In: $5^{\circ}$ ECAECO Encontro Científico de Administração, Economia e Contabilidade, 2012, Ponta Porã - MS. Desenvolvimento Regional, Inovação e Agronegócio, 2012.

KROGH, G., NONAKA, I.; RECHSTEINER, L.. Leadership in Organizational Knowledge Creation: A Review and Framework. Journal of Management Studies, v. 49, n.1, p. 240-277, 2012.

KUPERS, W. Inter-Place: Phenomenology of embodied space and place as basis for a relational understanding of leader and follow ship in organisations. Environment Space Place, v. 2, n. 1, p. 79-120. Zeta books, 2010. 
LANE, P.; LUBATKIN, M. Relative Absorptive Capacity and Interorganizational Learning. Strategic Management Journal, v. 19, n. 5, p. 461-477, 1998.

LARSSON, R., BENGTSSON, L., HENRIKSSON, K., SPARKS, J. The interorganizational learning dilemma: collective knowledge development in strategic alliances. Organization Science, v. 9, n. 3, p. 285-305, 1998.

LEVINSON, N.; ASAHI, M. Cross-National Alliances and Interorganizational Learning. Organizational Dynamics, New York, v. 24, n. 2, p. 50-64, 1995.

LUBATKIN, M.; FLORIN, J.; LANE, P. Learning together and Apart: A Model of Reciprocal Interfirm Learning. Human Relations, v. 54, n. 10, p. 1353-1382, 2001.

LUEN, T. W.; AL-HAWAMDEH, S. Knowledge Management in the Public Sector: Principles \& Practices in Police Work, Journal of Information Science, Vol. 27, pp. 311-318, 2001.

LUI, S.S. The Roles of Competence Trust, Formal Contract,; Time Horizon in Interorganizational Learning, Organization Studies, v. 30; p. 333-353, 2009.

MCCOLE, The role of trust for electronic commerce in services, International Journal of Contemporary Hospitality Management, v. 14, n. 2, pp. 81-87, 2002.

MELLAT-PARAST, M.; DIGMAN, L.A. A framework for quality management practices in strategic aliances, Management Decision, v. 45, n. 4, p. 802-818, 2007.

MOHR, J. SENGUPTA, S. Managing the paradox of Inter-firm: the role of governance Mechanisms. The Journal of Business \& Industrial Marketing, v. 17, n. 4, p. 282-301, 2002.

MÖLLER, K.; RAJALA, A. Rise of strategic nets - New modes of value creation, Industrial Marketing Management, v. 36, n. 7, p. 895-908, 2007.

MUTHUSAMY, S.K.; WHITE, M.A. Learning; Knowledge Transfer in Strategic Alliances: A Social Exchange View. Organization Studies, v. 26, n. 3, p. 415-441, 2005.

NONAKA, I. A dynamic theory of organizatinal knowledge creation Organization Science. Vol. 5, n. 1, fevereiro, 1994. 
Confiança, aprendizagem e conhecimento nos relacionamentos interorganizacionais: diagnóstico e análise dos avanços sobre o tema

, TAKEUCHI, H. Criação do Conhecimento na Empresa: como as empresas geram a dinâmica da inovação. Rio de Janeiro: Campus, 1997.

NOOTEBOOM, B. Inter-Firm Alliances. Analysis; Design, Routledge, London - New York, 1999.

OWEN-SMITH, J.; POWELL, W. W. Knowledge networks as channels and conduits: the effects of spillovers in the boston biotechnology. Organization Science, v. 15, n.1, p. 6-21, 2004.

PARK, B., WHITELOCK, J.; GIROUD, A. Acquisition of marketing knowledge in small; medium-sized IJVs: The role of compatibility between parents. Management Decision, v. 47, n. 8, p. 1340-1356, 2009.

PESÄMAA, O.; HAIR, J. F. More than friendship is required: An empirical test of cooperative firm strategies, Management Decision, v. 45, n. 3, p. 602-615, 2007.

PROVAN, K. G.; FISH, A.; SYDOW, J. Interorganizational Networks at the Network Level: A Review of the Empirical Literature on Whole Networks. Journal of Management, June 2007, v. 33, p. 479-516, 2007.

RUIZ-MERCADER, S.J; MERONO-CERDAN A.L.; SABATER-SANCHEZ, R. Information technology and learning: Their relationship and impact on organizational performance in small businesses. International Journal of Information Management, v. 26, p. 16-29, 2006.

SAILER, R. E. Structural equivalence: meaning; definition, computation; application. Social Networks, v. 1, n. 1, p. 73-90, 1978.

SAMADDAR, S.; KADIYALA, S. S. An analysis of interorganizational resource sharing decisions in collaborative knowledge creation. European Journal of Operational Research, v. $170,2006$.

SCARBROUGH, H. Knowledge management, HRM; innovation process, International Journal of Manpower, v. 24, n. 5, p. 501-516, 2003. 
SCHREINER, M.; KALE, P.; CORSTEN, D. What really is alliance management capability and how does it impact alliance outcomes and success? Strategic Management Journal, forthcoming, 2009.

SMITH, D. A.; LOHRKE, F. T. Entrepreneurial network development: Trusting in the process, Journal of Business Research, v. 61; p. 315-322, 2008.

SCHILDT, H.; KEIL, T.; MAULA, M. The temporal effects of relative and firm-level absorptive capacity on interorganizational learning. Strategic Management Journal, v., 33, p.1154-1173, 2012.

SCHULTZE, U.; LEIDNER, D.E. Studying knowledge management in information systems research: discourses and theoretical assumptions. MIS Quarterly, v,. 26, n. 3, p. 213-242, 2002.

STEPHENS, K. J., FULK, J.; MONGE, P. Constrained choices in alliance formations: Cupids; organizational marriages, Humans Relations, v. 62, n. 4, p. 501-536, 2009.

SWAN, J. E.; NOLAN, J. J. Gaining customer trust: a conceptual guide for the sales-person. Journal Personal Selling Sales Manage, v. 5, p. 39-48, 1985.

SWAN, J. E.; TRAWICK, I. F. Jr. Building Customer Trust in the Industrial Salesperson: Process and Outcomes. Advance in Business Marketing, v. 2, p. 81-113, 1987.

TAGLIAVENTI, M.R.; MATTARELLI, E. The Role of Networks of Practice, Value Sharing, and Operatioanl Proximity in Knowledge Flows Between Professional Groups. Human Relations, v. 59, n. 3, p. 291-319, 2006.

TANG, F.; MU, J.; MACLACHLAN, D. L. Implication of network size and structure on organizations knowledge transfer. Expert Systems with Applications, v. 34, n. 2, p. 11091114, 2008.

TANRIVERDI, H.; VENKATRAMAN, N. Knowledge relatedness and performance of multibusiness firms. Strategic Management Journal, v. 26, p. 97-119, 2005.

TEECE, D. J. The Multinational Corporation and the Resource Cost of International Technology Transfer. Ballinger, Cambridge, MA, 1976.

REAd | Porto Alegre - Edição 76 - N 3 - setembro/dezembro 2013 - p. 709-737 
Confiança, aprendizagem e conhecimento nos relacionamentos interorganizacionais: diagnóstico e análise dos avanços sobre o tema

. Transactions cost economics and the multinational enterprise', Journal of Economic Behavior and Organization, v. 7, p. 21-45, 1986.

.Firm organization, industrial structure, and technological innovation', Journal of Economic Behavior and Organization, 31, pp. 193-224, 1996.

.; GARY, P.; AMY, S. Dynamic capabilities and strategic management, Strategic Management Journal, v. 18, n. 7, p. 509-533, 1997.

THAU, S., CROSSLEY, R., BENNETT, J.; SCZESNY, S. The relationship between trust, attachment, antisocial work behaviors, Human Relations, v. 60, n. 8, p.1155-1179, 2007.

URSO, G. et al., Knowledge Sharing is Power. Transition Studies Review, v. 16, p. 2, p. 352$367,2009$.

UZZI, B. Social Structure and Competition in interfirm Networks: The paradox of embeddeness. Administrive Science Quartely, v. 42, p. 35-67, 1997.

WAI-KIT NG, P., LAU, C.M.; NYAW, M.K. The effect of trust on international joint venture performance in China, Journal of International Management, v. 13; p. 430-448, 2007.

WALKER, D. H. T.; CHRISTENSON, D. Knowledge wisdom and networks: a project management centre of excellence example, The Learning Organization, vol. 12, no. 3, p. 275, 2005 .

WANG, S.; NOE, R. A. Knowledge sharing: A review and directions for future research. Human Resource Management Review, v. 20, n. 2, p. 115-131, 2010.

WILLEM, A.; BUELENS, M. Knowledge sharing in inter-unit cooperative episodes. The impact of organizational structure dimensions. International Journal of Information Management. v. 29, n. 2, p. 151-160, 2009.

WILLIAMSON, O. E. Market and Hierarchies: Analysis and Antitrust Implications . New York: The Free Press, 1975. 
Juliano Nunes Alves \& Breno Augusto Diniz Pereira

ZAHEER, A.; VENKATRAMAN, N. Relational governance as an interorganizational strategy: An empirical test of the role of trust in economic exchange. Strategic Management Journal. v. 16; p. 373-392, 1995.

ZAHEER, A.; MCEVILY, B; PERRONE, V. Does Trust Matter? Exploring the Effect Interorganizational; Interpersonaltrust on Performance, Organization Science, v. 9, n. 2, p. 141-159, 1998.

ZAIDMAN, N.; BROCK, D. M. Knowledge transfer within multinationals and their foreign subsidiaries: A culture-context approach. Group \& Organization Management, v. 34, n. 3, p. 297-329, 2009.

ZARVANDI, N.; ZARVANDI, J. Conceptualizations of Trust in the Organization. Ideal Type of Management, v. 1, n. 1, p. 37-46, 2012.

ZHAO, Z.; ANAND, J.; MITCHELL, W. 2005. A Dual Networks Perspective on InterOrganizational Transfer of R\&D Capabilities: International Joint Ventures in the Chinese Automotive Industry. Journal of Management Studies, v. 42, n. 1, p. 127-160. 